\section{Influence of antenna configurations on performance of STBC in urban microcells}

\section{J. Salo, B. Badic, P. Suvikunnas, H. Weinrichter, M. Rupp} and P. Vainikainen

Based on urban microcell channel measurements, the bit error rate performance of a space-time block code with four transmit antennas using various dual-branch receive antenna configurations, is evaluated. It is demonstrated that with realistic handset antennas, it is possible to achieve performance very close to that of the theoretical uncorrelated Rayleigh case, although proximity of operator tissue (e.g. head) will result in several decibels performance degradation.

Introduction: The performance of coded data transmission over multiple-input multiple-output (MIMO) channels depends on the spatial characteristics of antennas and the propagation environment. Even so, it is typically evaluated using idealised channel models, that are unable to capture the combined effect of real-world radio channels and handset antennas on wireless link performance. The three main factors degrading performance of real-world MIMO communications systems are spatial correlation, channel coefficient power imbalance, and SNR degradation owing to unfavourable antenna orientation. In particular, field patterns of realistic mobile handset antennas are not omnidirectional, and the received signal typically arrives from few dominating directions, or angular clusters. As a consequence, directivity and orientation of the antenna patterns play an important role in estimating system performance. In this Letter, we evaluate the effect of antennas on the bit error ratio (BER) performance of a quasiorthogonal space-time block code (QSTBC) for four transmit antennas in a practical urban microcell scenario.

Channel measurements: Measurements were conducted in downtown Helsinki at $2.1 \mathrm{GHz}$ carrier frequency. The transmitter had two dualpolarised patch antennas (four tx channels) with $45 \mathrm{~cm}$ (three wavelengths) element separation. The receiver was a spherical antenna with 32 dual-polarised elements. The receiver and the transmitter heights were 1.5 and $13 \mathrm{~m}$, respectively. The height of the surrounding buildings was $15-25 \mathrm{~m}$. This is a typical urban microcell scenario. The measurement system and the environment have been explained in more detail in [1]. The receiver moved in non-line-of-sight (NLOS) scenario $60 \mathrm{~m}$ along a street perpendicular to the line-of-sight street at $0.4 \mathrm{~m} / \mathrm{s}$ speed (from $50 \mathrm{~m}$ point to the end of the route 'Rout' in [1, Figs. 2a and 6a]). The time-variant channel matrix was measured four times per wavelength. The total number of channel instances recorded was $N=1696$.

Data processing: The direction-of-arrival, amplitude, and polarisation of the plane waves impinging on the spherical receive array were estimated with a beamforming based method [1]. The plane wave representation of the received signal was combined with the simulated dual-polarised 3D radiation patterns of the handset antennas using the measurement-based antenna test bed (MEBAT) [2]. To examine the effect of antenna orientation, antenna radiation patterns were rotated to $L=8$ different 'look directions' ( $45^{\circ}$ azimuth grid). Hence, a total of $N L=13568$ channel matrices were obtained for the BER evaluation. To apply correct SNR in the BER simulations, large-scale fading, i.e. shadowing and path loss, was estimated from data with wavelet regression using Donoho's threshold [3]. In computations, we applied eight samples (two wavelengths) pre-smoothing, followed by a wavelet denoising step using the Matlab's 'wden' function and eighth-order symlet with third-level decomposition. We denote the $n$th realisation of the $n_{r} \times n_{t}$ channel matrix with $\mathbf{H}_{n}^{l}$, and its $(i, j)$ th element with $h_{i j, n}^{l}$; superscript $l$ denotes the antenna orientation $(l=1, \ldots, L)$. Sample mean over index $x$ is denoted with ave ${ }_{x}[\cdot]$. The following three normalisation methods were employed to normalise the channel matrices. M1: large-scale fading was removed from each channel coefficient $\left\{\left|h_{i j, n}^{l}\right|\right\}_{n=1}^{N}$ separately, and each channel coefficient was scaled to unit mean power so that ave $_{n}\left[\left|h_{i j, n}^{l}\right|^{2}\right]=1$ for all $i, j, l$. M2: large-scale fading was removed from the sequence of Frobenius norms $\left\{\left\|\mathbf{H}_{n}^{l}\right\|_{F}\right\}_{n=1}^{N}$, and matrix power was normalised so that $\operatorname{ave}_{n}\left[\left\|\mathbf{H}_{n}^{l}\right\|_{F}^{2}\right]=n_{r} n_{t}$ for all $l$. M3: large-scale fading was removed from the signal power averaged over all antenna orientations, i.e. from a sequence of squared Frobenius norms $\left\{P_{n}\right\}_{n=1}^{N}$, where

$P_{n}=\operatorname{ave}_{l}\left[\left\|\mathbf{H}_{n}^{l}\right\|_{F}^{2}\right]$. The channel matrices were normalised over all $N$ observations so that $\operatorname{ave}_{n}\left(P_{n}\right)=n_{r} n_{t}$. Comparing BER results between methods M1 and M2 reveals the effect of channel coefficient power imbalance, whereas comparing BER results between M2 and M3 reveals the effect of antenna orientation on the SNR. M3 is the most realistic normalisation and will be used to predict BER performance under real-world conditions.

Antennas: In this study we use a total of five antenna patterns: three synthetic reference patterns, and two realistic handset antenna patterns. We assume, for simplicity, that all antennas have ideal efficiencies. The synthetic patterns are as follows (Fig. 1):

-'iso': The element patterns are isotropic. The gain of $\mathrm{H}$ and $\mathrm{V}$ polarisations is $2^{-0.5}$. The element separation is 0.3 wavelengths $(5 \mathrm{~cm}$ at $2.1 \mathrm{GHz})$.

-'dir1': The directivities of the receiver branches are proportional to $[\sin (\theta) \cos (\phi)]^{n}$, where $\theta \in\left[0^{\circ}, 180^{\circ}\right]$ is the elevation angle, $\phi \in\left[-90^{\circ}\right.$, $\left.90^{\circ}\right]$ is the azimuth angle, and $n=1.57$. The antenna gain in this case is about $7 \mathrm{dBi}$ with half power beamwidth of about $100^{\circ}$, in both azimuth and elevation. The polarisation is vertical with ideal cross-polarisation discrimination.

-'dir2': The element patterns are as with 'dir1', but the field patterns of the receiver branches point to the same direction.

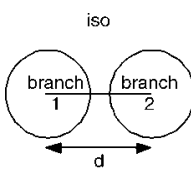

high spatial correlation for small d

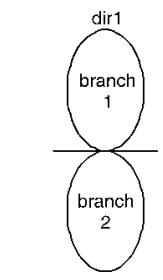
to antenna orientation high branch power imbalance
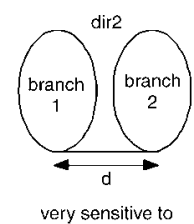

antenna orientation

high spatial high spatial
correlation for small c$$
\text { (1) }
$$

Fig. 1 Synthetic radiation patterns and main drawbacks (in simulations, $d=0.3 \lambda$ used)

Mutual coupling effects are neglected with the synthetic patterns. We also evaluate the BER performance with a realistic handset-internal dual-branch antenna 'A3' introduced in [4]. The radiation pattern of 'A3' was also simulated with an electromagnetic model of a human head placed next to the antenna [4]. We abbreviate this configuration as 'A3h'. The effect of the head is to increase the directivity of the antenna patterns. Due to space limitations we refer to [4] for details of 'A3' and 'A $3 \mathrm{~h}$ '.

Space-time block code: We use the QSTBC for four transmit antennas introduced in [5]. The code has rate-1, good performance at low to medium SNRs, and a simple decoding algorithm. QPSK modulation is used in all cases. Perfect channel estimation and maximumlikelihood decoding are assumed at the receiver.

Spatial correlation and power imbalance: Envelope correlation values between channel matrix elements were computed from channel matrices normalised with method M1. To reduce the amount of data we retain only the worst-case correlation coefficients for each antenna orientation, i.e. $c^{l}=\max \left(\mathbf{C}^{l}\right)$, where the maximum is taken over all off-diagonal elements of the full $8 \times 8$ covariance matrix $\mathbf{C}^{l}$. The maximum, minimum and average over antenna orientations are reported in Table 1. In Table 1 we also report the imbalances in average powers between receiver branches. We denote $\delta^{l}=\left(P_{\min }^{l}\right)^{-1} P_{\max }^{l}$, where $P_{\min }^{l}$ and $P_{\text {max }}^{l}$ are the average power of the weaker and stronger receiver branch, respectively, evaluated over the measurement route for a fixed orientation $l$. Note that since the elements of the transmit array see the same propagation environment, the power imbalances occur mainly between the receiver branches. The results in Table 1 confirm the intuition that the directional patterns pointing in opposite directions ('dir1') have low spatial correlation but high branch power imbalance, which, however, degrades the BER performance of 'dir1' only about $1.2 \mathrm{~dB}$ at $\mathrm{BER}=10^{-4}$ (not shown). In contrast, pointing the patterns to the same direction ('dir2') increases spatial correlation, but reduces 
power imbalance. The branch patterns of realistic antennas 'A3' and 'A $3 \mathrm{~h}$ ' have the worst-case correlation coefficient of 0.6 , which is reasonably low from the diversity performance point of view. Over the practically important range of $\mathrm{BER}=10^{-2}-10^{-4}$, the effect of spatial correlation is small with all examined antennas.

Table 1: Envelope correlations $\left(c^{l}\right)$ and power imbalances $\left(\delta^{l}\right)$ for different antennas. Max, min, ave taken over antenna orientations

\begin{tabular}{|c|c|c|c|c|c|c|}
\hline Ant. & $\max _{l}\left(c^{l}\right)$ & $\min _{l}\left(c^{l}\right)$ & $\operatorname{ave}_{l}\left(c^{l}\right)$ & $\max _{l}\left(\delta^{l}\right)$ & $\min _{l}\left(\delta^{l}\right)$ & $\operatorname{ave}_{l}\left(\delta^{l}\right)$ \\
\hline iso & 0.80 & 0.59 & 0.64 & $0.1 \mathrm{~dB}$ & $0 \mathrm{~dB}$ & $0.1 \mathrm{~dB}$ \\
\hline dir1 & 0.11 & 0.08 & 0.09 & $12 \mathrm{~dB}$ & $1.1 \mathrm{~dB}$ & $9.5 \mathrm{~dB}$ \\
\hline dir2 & 0.88 & 0.51 & 0.72 & $0.5 \mathrm{~dB}$ & $0 \mathrm{~dB}$ & $0.2 \mathrm{~dB}$ \\
\hline A3 & 0.60 & 0.20 & 0.49 & $4.6 \mathrm{~dB}$ & $0.7 \mathrm{~dB}$ & $2.4 \mathrm{~dB}$ \\
\hline A3 h & 0.60 & 0.32 & 0.47 & $1.8 \mathrm{~dB}$ & $0.2 \mathrm{~dB}$ & $0.8 \mathrm{~dB}$ \\
\hline
\end{tabular}

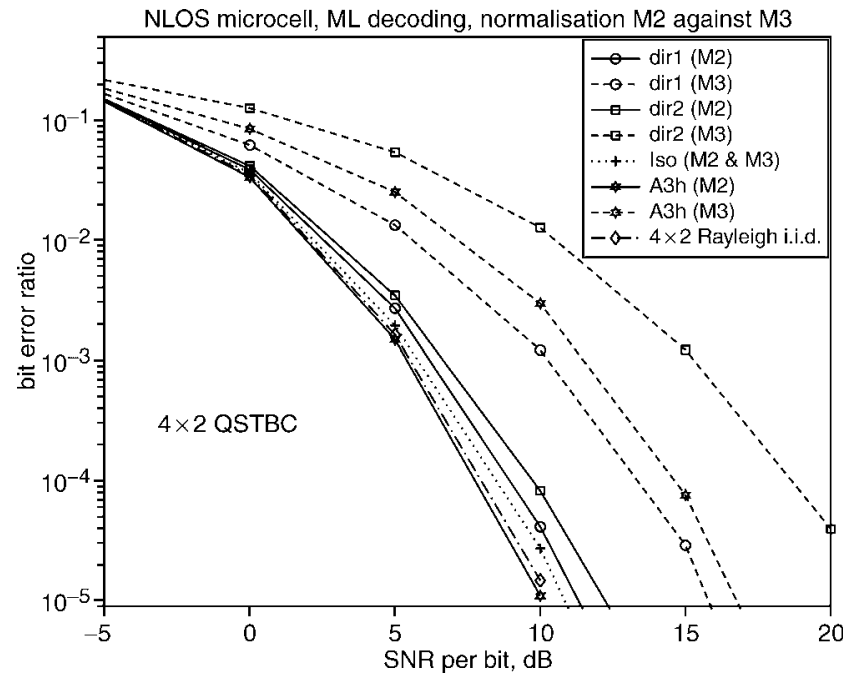

Fig. 2 Antenna orientation and BER

Normalisation M2 eliminates average power differences between antenna orientations, whereas $\mathrm{M} 3$ retains them

Antenna orientation: Fig. 2 illustrates the effect of antenna orientation on the BER performance. Applying normalisation method M2, antenna orientation has no effect on the mean received power, whereas with method M3 the power differences are retained. With directional patterns ('dir1', 'dir2', 'A3h') the BER increases dramatically, since the directions of arrival of the received signal are not uniform over the azimuth, and consequently the SNR fluctuates heavily about its mean depending on the antenna orientation. For the isotropic pattern antenna orientation has no effect. Interestingly, the performance of 'A3' with M2 normalisation is practically identical (not shown) with the Rayleigh i.i.d. channel; the degradation owing to antenna orientation (M3) is less than $1 \mathrm{~dB}$. The result suggests that, in free space conditions, it is possible to achieve performance very close to that of Rayleigh i.i.d. channels, even with realistic handset antennas. However, the proximity of user's head ('A3h') degrades receiver performance more than $4 \mathrm{~dB}$ owing to increased directivity and, consequently, sensitivity to antenna orientation. In reality, this degradation would be even larger because of reduced radiation efficiency.

Conclusions: Using measured channel matrices, we have evaluated the effect of spatial correlation, channel power imbalance and antenna orientation on the BER performance of a QSTBC. With directional patterns, antenna orientation has a strong impact on SNR, and hence the BER. Spatial correlation and receiver branch power imbalance were found to have a minor effect, even for closely spaced antennas. Our results indicate that with realistic handset antennas in free space conditions, BER performance close to that of the uncorrelated Rayleigh case can be achieved. As a general design rule, the signal power received by an antenna should be insensitive to antenna orientation and therefore one should aim to minimise the directivity of the patterns.

Acknowledgment: We appreciate J. Ollikainen's clever handset antenna designs.

\section{(C) IEE 2005}

Electronics Letters online no: 20052904

9 August 2005 doi: 10.1049/el:20052904

J. Salo, P. Suvikunnas and P. Vainikainen (DC SMARAD/Radio Laboratory, Helsinki University of Technology, P.O. Box 3000, FI02015-TKK, Finland)

E-mail: jari.salo@tkk.fi

B. Badic, H. Weinrichter and M. Rupp (Institute of Communications and Radio Frequency Engineering, Vienna University of Technology, Gußhausstraße 25/389, A-1040 Vienna, Austria)

\section{References}

1 Sulonen, K., Suvikunnas, P., Vuokko, L., Kivinen, J., and Vainikainen, P.: 'Comparison of MIMO antenna configurations in picocell and microcell environments', IEEE J. Sel. Areas Commun., 2003, 21, (5), pp. 703-712

2 Suvikunnas, P., Sulonen, K., Villanen, J., Icheln, C., and Vainikainen, P.: 'Evaluation of performance of multiantenna terminals using two approaches'. Proc. IEEE Instrumentation and Measurement Tech. Conf., Lake Como, Italy, May 2004, Vol. 2, pp. 1091-1096

3 Donoho, D.L., and Johnstone, I.M.: 'Ideal spatial adaptation by wavelet shrinkage', Biometrika, 1994, 81, pp. 425-455

4 Villanen, J., Suvikunnas, P., Sulonen, K., Ollikainen, J., Icheln, C., and Vainikainen, P.: 'Advances in diversity performance analysis of mobile terminal antennas'. Proc. Int. Symp. Antenna Propagation., Sendai, Japan, Augst 2004, pp. 649-652

5 Jafarkhani, H.: 'A quasi-orthogonal space-time block code', IEEE Trans. Commun., 2001, 49, (1), pp. 1-4 